\title{
Type Inference for Action Semantics
}

\author{
Susan Even* and David A. Schmidt ${ }^{\dagger}$ \\ Computing and Information Sciences Dept. \\ Kansas State University, Manhattan, KS 66506 USA \\ schmidt@cis.ksu.edu
}

\section{Introduction}

In a series of papers $[11,12,13,14,15,25,26]$, Mosses and Watt define action semantics, a metalanguage for high level, domain-independent formulation of denotational semantics definitions. Action semantics was designed to support readability, abstraction, modularity, and modifiability of language definitions $[13,15]$.

In our recent work [5], we studied a combinator-based version of action semantics. We developed a typing system for actions based on types and kinds. The types within a kind are partially ordered to refiect subtyping. We then defined a category-sorted algebra model $[18,20]$ for the system where actions are natural transformations over interpretation functors (which map the type names and their ordering to predomains and coercion functions). Thus, an action is a family of continuous functions that behave consistently with respect to the subtyping relation. Coercion functions- even nonembeddings- disappear.

The typing system and its interpretation support an ML-style type inference algorithm for action expressions. Type inference must respect the subtyping laws, so we encounter the problems discovered by Mitchell [10], Fuh and Mishra [6], and Wand [24] regarding constraints sets. But action notation and its model are formulated so that the major problems regarding constraints are "restricted away." In particular, the monotonic behavior of actions with respect to the subtyping ordering keeps the cardinality of constraints sets small and makes constraints on record types unnecessary, even in the presence of record union and concatenation operations.

This paper presents our variant of action semantics and its typing system. We give the type inference algorithms, state properties that action semantics expressions satisfy, give soundness and completeness properties and explain why they hold for type inference on action semantics expressions. We also present a small example.

\section{Action Semantics}

In our version of action semantics, actions are combinators that operate upon facets (also called kinds) [11,14]. A facet is a collection of types; for example, the functional facet is the kind of all types that can be used as temporary values ("transient information" [14]) in a computation. The types int, bool, real, bool xreal, and so on, belong to the functional facet. The other facet studied in this paper is the declarative facet, which contains types of identifier, value binding ("scoped information"). The types in this facet are record types [3,19]. Two other facets, the imperative facet and the communicative facet [14], are omitted for brevity's sake.

The types in a facet are partially ordered to reflect subtyping relationships $[2,18,19]$. For example, the type int is a subtype of the type real, written int $\leq$ real $[3,19]$. Values of type int may be used in any context in which a real may appear. Figure 1 lists the types in the functional and declarative facets and their orderings. We use ns ("nonsense") to stand for an undefined type. Call ns the

* Partially supported by ONR Grant N-00014-88-K0455. † Partially supported by NSF Grant CCR-8822378. 
Functional facet: Types are Proper-functional-type $\cup$ \{ ns \}, where:

$t \in$ Proper-functional-type

$p \in$ Primitive-type

$$
\begin{aligned}
& t:=p\left|t_{1} \rightarrow t_{2}\right| t_{1} \times t_{2} \\
& p:=\text { int } \mid \text { real } \mid \text { bool } \mid \cdots
\end{aligned}
$$

The ordering is the smallest reflexive, transitive ordering such that:

int $\leq$ real, etc., on the primitive-types

$t \leq \mathrm{ns}$ for all $t$

$t_{1} \rightarrow t_{2} \leq t_{1}{ }^{\prime} \rightarrow t_{2}{ }^{\prime}$ iff $t_{1}{ }^{\prime} \leq t_{1}$ and $t_{2} \leq t_{2}{ }^{\prime}$

$t_{1} \times t_{2} \leq t_{1} \times t_{2}^{\prime}$ iff $t_{1} \leq t_{1}{ }^{\prime}$ and $t_{2} \leq t_{2}{ }^{\prime}$

Declarative facet: Types are Proper-declarative-type $\cup$ [ns ], where:

deProper-declarative-type

$$
\begin{aligned}
d::= & \left\{i: t_{i}\right\}_{i \in I} \mid\left\{i: t_{i}\right\}_{i \in I \text { exactly }} \\
& \text { where } I \text { is a finite set of identifiers }
\end{aligned}
$$

The ordering is the smallest reflexive, transitive ordering such that:

$d \leq \mathrm{ns}$ for all $d$

$\left\{i: t_{i}\right\}_{i \in I} \leq\left\{j: t_{j}{ }^{\prime}\right\}_{j \in J}$ iff $J \subseteq I$ and for all $j \in J, t_{j} \leq t_{j}{ }^{\prime}$

$\left\{i: t_{i}\right\}_{i \in I \text { exactly }} \leq\left\{i: t_{i}\right\}_{i \in I}$

$\left\{i: t_{i}\right\}_{i \in I \text { exacty }} \leq\left[i: t_{i}{ }^{\prime}\right\}_{i \in I \text { exactly }}$ iff for all $i \in I, t_{i} \leq t_{i}{ }^{\prime}$

improper type; all other types are proper types.

The types for records deserve closer scrutiny. It is customary to read the type $\left\{i: t_{i}\right\}_{i \in I}$ as the type of records that have at least bindings of type $t_{i}$ for identifiers $i \in I$ (but may have additional bindings) $[1,3]$ and the type $\left\{i: t_{i}\right\}_{i \in I \text { exectly }}$ as the type of records that have bindings for exactly the identifiers in $I$ and no others. But the key to understanding the two forms of record type is the subtyping ordering on them: the $\left\{i: t_{i}\right\}_{i \in I}$-types use the "inheritance subtyping" of [3], whereas the $\left\{i: t_{i}\right\}_{i \in I \text { exactly }}$-types do not. We have found good use for the two forms of record types: some actions operate well under inheritance subtyping and some simply do not. We can accommodate both.

Figure 2 gives the interpretations for the proper types. Note that we naively interpret the types $\left\{i: t_{i}\right\}_{i \in I}$ and $\left\{i: t_{i}\right\}_{i \in I \text { exactly }}$ the same way! This is surprising in light of the previous paragraph, but we wish to emphasize that the subtyping ordering is the crucial feature of records. The ordering is interpreted into coercion functions on the interpreted types. Since the coercions need not be mere embeddings [18], we may well be concerned about the computational overhead in using them. But we will soon see that coercions are never explicitly or implicitly inserted in action expressions.

The improper type, ns, is interpreted as the singleton set; a coercion into ns is the constant function.

The interpretation of the types and subtyping relation is formalized as a functor from the partial ordering of type names, treated as a category, into the category Pdom of predomains $[18,20]$.

Actions are mappings whose domains and codomains are facets. We call the domain of an action its source, and its codomain its target. For example, we write copy: $F \rightarrow F$ to state that the source and target of action copy are the functional facet (" $F$ "). (The declarative facet will be represented by a " $D$ ".) Since a facet contains many types, actions are polymorphic mappings on the predomains that 
Figure 2

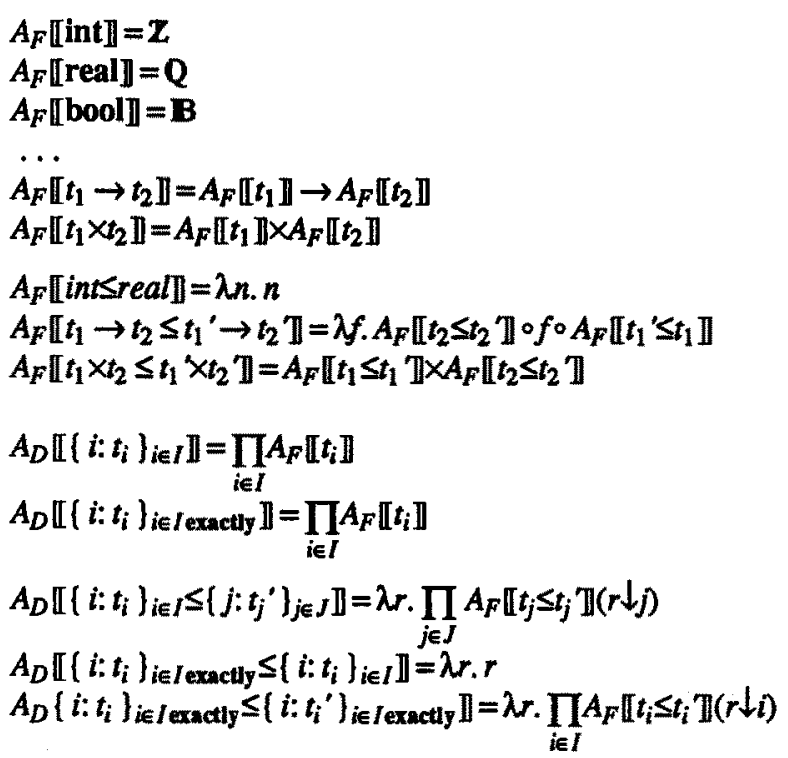

the types denote. Each action $a$ has a monotonic typing function $T_{a}$ that describes its behavior on argument types. For example, copy is the identity mapping on the functional facet, so its typing function is $T_{\text {copy }}=(\lambda t: F . t)$, which states that copy maps an argument of type $t$ to an answer of type $t$. The meaning of copy is the family of identity functions $\{\lambda v: t . v\}_{t \in F}$, which we represent by $\lambda t: F . \lambda v: t . v$.

Actions exist for all the fundamental operations of programming languages: value passing, arithmetic, binding creation and lookup, storage allocation and updating, and so on [11,13,15]. Figure 3 presents a minimal subset of primitive actions that is representative of action sets for modelling statically typed languages. We have already seen copy; succ increments arguments from int and real and is undefined on nonnumbers; put $v$ emits value $v$ as its result; pass is the identity mapping for the declarative facet; bind $i$ emits a declarative facet record that holds exactly the one binding of identifier $i$ to the argument received by the action; find $i$ retrieves from its declarative record argument the binding to $i$; forget $t_{D}$ takes arguments from the two facets and outputs the one from the functional facet;

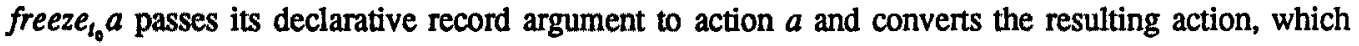
awaits a functional facet value, into a functional facet value itself and outputs it; and eval applies a function to an argument.

The third column of Figure 3 states that an action $a: K_{1} \rightarrow K_{2}$ is a family of mappings $\left\{f_{k}: k \rightarrow T_{a}(k)\right\}_{k \in K_{1}}$ that behave naturally, that is, it respects the subtyping ordering:

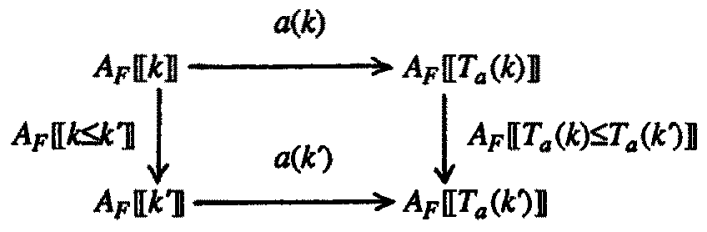


Figure 3

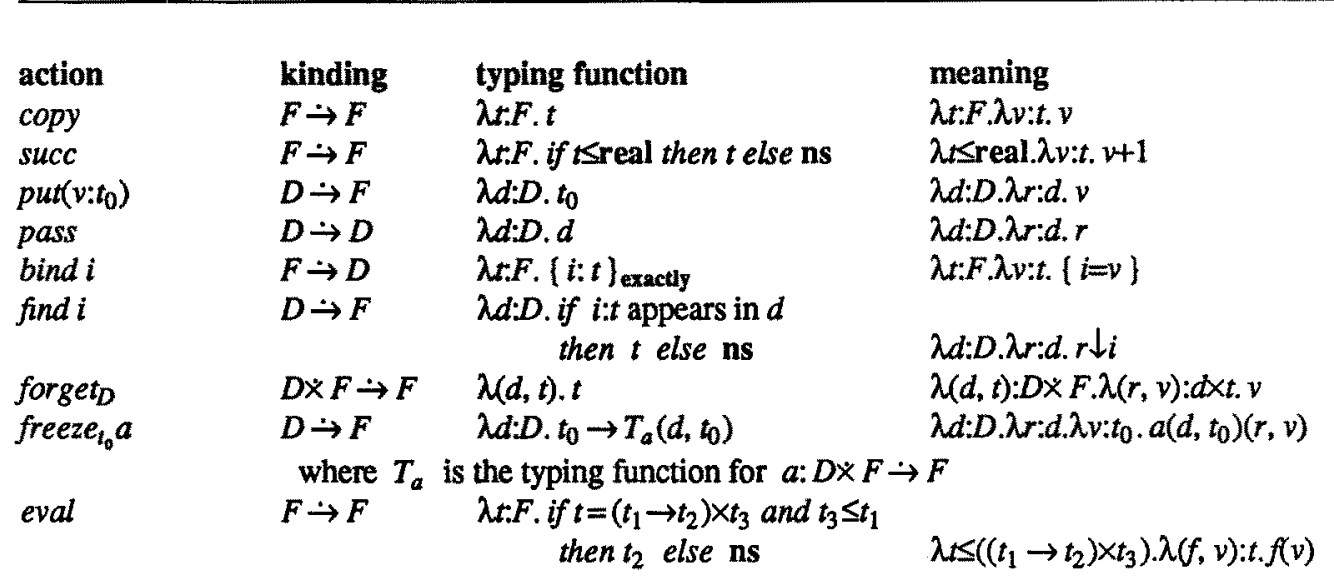

(note: all typing functions and meanings are "ns-strict": for action $a, T_{a}(\mathrm{~ns})=n s$ and $a(\mathrm{~ns})=\lambda 0.0$ )

where $k, k^{\prime} \in K_{1}$ and $k \leq k^{\prime}$. Thus, an action $a$ is a natural transformation from the functor $A_{F}$ to the functor $A_{F} \circ T_{a}[18,20]$.

Modelling actions as natural transformations has several important consequences. The first is that coercion functions - even nonembedding coercions - need not be inserted into a composite natural transformation. The commutativity property, shown above, of a natural transformation guarantees that the answer produced by a composite natural transformation from an argument must be unique regardless of what coercions (if any) are applied to the argument, the answer, or the intermediate values produced by components of the composite natural transformation. So, we don't use coercions.

The second consequence is that the interpretation of a record type $\left\{i: t_{i}\right\}_{i \in I}$ is not critical. The essence of inheritance typing is expressed in the subtyping ordering and the respect that a natural transformation has for the ordering. An argument of type $\left\{i: t_{i}\right\}_{i \in I \text { exactly }}$ must be treated consistently by a natural transformation as if it also belonged to those record types $\left\{j: t_{j}{ }^{\prime}\right\}_{j \in J}$, such that $J \subseteq I$, $t_{j} \leq t_{j}, j \in J$. But as the previous paragraph noted, the argument need never be coerced into $\left\{j: t_{j}{ }^{\prime}\right\}_{j \in J}$. At first glance, none of the actions in Figure 3 appear to exploit inheritance typing. But actions like find $i$ are forced to have uniform structure because of it. Also, there exist actions analogous to freeze $_{t_{0}} a$ and eval that operate on the declarative facet and make crucial use of inheritance typing.

Finally, the modelling of actions as natural transformations prevents self-application. This justifies the definition of the action freeze $t_{0} a$. For an argument $d_{0} \in D$, freeze $t_{t_{0}} a$ must output action $a$ bound to $d_{0}$. But this is impossible, for such a result would be an action (natural transformation) of kinding $F \rightarrow F$. So, the output must be the functional facet value $a\left(d_{0}, t_{0}\right)$, a function of type $t_{0} \rightarrow T_{a}\left(d_{0}, t_{0}\right)$, instead. The eval action, which activates $a\left(d_{0}, t_{0}\right)$, exploits the subtyping ordering to recover (some of) the polymorphism in $a$.

We study three forms of action composition: $a ; a^{\prime}$, sequential composition; $a * a^{\prime}$, parallel composition; and $\triangleright a$, a restricted concatentation composition. Figure 4 shows the flow of arguments directed by the compositions.

The composition $a_{1} ; a_{2}$ represents sequential composition: arguments to $a_{1} ; a_{2}$ are given to $a_{1}$, which produces results that are given to $a_{2}$. The results produced by $a_{2}$ are the results for the composed action. The target of $a_{1}$ must equal the source of $a_{2}$. For example, copy; succ accepts a functional facet value that is passed sequentially from copy to succ, and the output is the incremented value. 


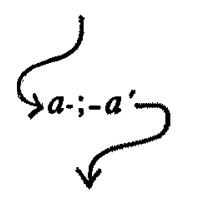

sequential

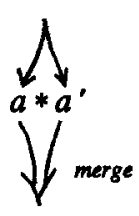

parallel

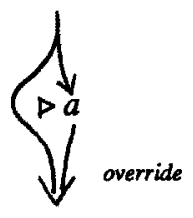

concatenation

The composition $a_{1} * a_{2}$ represents parallel composition: arguments to $a_{1} * a_{2}$ are given to both $a_{1}$ and $a_{2}$, each of which produces its own results. The results are merged to produce the results for the composed action. (In the functional facet, merging is tupling; in the declarative facet, merging is record union.) The sources of $a_{1}$ and $a_{2}$ must be identical. For example, copy*succ accepts an argument, which is given to both copy and succ. The two results - the value and its successor- are merged into a pair. As another example, the declarative record argument to (put 3 ; bind $A) *($ find $C$; bind $B$ ) is given to the two operands of *. The left operand ignores the record, emitting a binding of $A$ to 3 . The right operand finds the binding to $C$ and uses it to make a binding to $B$. The bindings to $A$ and $B$ are unioned into one declarative record.

The composition $>a$ represents concatenation composition: the declarative record argument to the action is given to $a$, and $a$ 's output, which is another declarative record, is concatenated to the original record, overriding bindings in the original argument. For example, $>$ (find $A$; bind $B$ ) accepts a record, which is used to make a binding of $B$ to $A$ 's value. The binding to $B$ is concatenated to the original bindings. (The form of concatenation composition given here is a restricted version of the binary composition $a \triangleright a^{\prime}$, where the outputs of the two actions are concatenated. Thus, the unary form is really pass $\triangleright a$, which explains why we treat $\triangleright a$ as a "composition.")

Figure 5 lists the compositions. The typing function $T_{\text {union }}$ limits record union so that only records with "exactly" types (i.e., types of form $\left\{i: t_{i}\right\}_{i \in / \text { exactly }}$ can be unioned. One way of understanding this restriction is that the record union operation can not union together two records with extra, "hidden" fields. (The union of a record of type [A:bool] to one of type [B:int] might fail because the first record might have a hidden B-binding that clashes with the B-binding in the second record.) But another way of understanding the restriction is that the record union operation simply does not behave well with inheritance subtyping. (A union of a record with just an A-binding to one with just a B-binding will succeed, but a union of a record with A- and B-bindings to one with a B-binding fails, even though the record with A- and B-bindings has a type that is a subtype of records with just A-bindings.) A similar problem arises with record concatenation, and $T_{\text {concat }}$ handles it in a similar way.

Figure 6 shows a sample language definition in action semantics.

\section{Type Inference of Action Expressions}

We might translate a source program through the semantics definition of Figure 6 and then type check the action semantics expression. For example, the typing scheme $\{A$ :present $(\theta)\} \alpha \rightarrow \theta$ if $\theta \leq$ real is the best typing scheme for the expression $\mathbf{E}\left[B=2:\right.$ int in add $A B \rrbracket=a_{0}: D \rightarrow F$, where: 
Figure 5

for $a: K_{1} \rightarrow K_{2}$ and $a^{\prime}: K_{1}^{\prime} \rightarrow K_{2}^{\prime}$

action kinding

$a ; a^{\prime} \quad K_{1} \rightarrow K_{2}^{\prime}$ if $K_{2}=K_{1}^{\prime}$

$a * a^{\prime} \quad K_{1} \rightarrow F$ if $K_{1}=K_{1}^{\prime}$

and $K_{2}=K_{2}{ }^{\prime}=F$

$K_{1} \rightarrow D$ if $K_{1}=K_{1}$ '

and $K_{2}=K_{2}{ }^{\prime}=D$

$\triangleright a \quad D \rightarrow D$ if $K_{1}=K_{2}=D$

$F \times D \rightarrow D$ if $K_{1}=F \times D$

$$
\text { and } K_{2}=D
$$

typing function

$T_{a} \circ T_{a}$

$\lambda k: K_{1}, T_{a}(k) \times T_{a^{\prime}}(k)$

$\lambda k: K_{1}, T_{\text {union }}\left(T_{a}(k), T_{a^{\prime}}(k)\right)$

$\lambda d: D . T_{\text {concat }}\left(d, T_{a}(d)\right)$

$\begin{aligned} & \lambda(t, d): F \times D . T_{\text {concat }}\left(d, T_{a}(t, d)\right) \\ & \lambda(t, d): F \times D . \lambda(v, r): t \times d . \operatorname{concat}(r, a(t, d)(v, r))\end{aligned}$

$\begin{aligned} & \lambda(t, d): F \times D . T_{\text {concat }}\left(d, T_{a}(t, d)\right) \\ & \lambda(t, d): F \times D . \lambda(v, r): t \times d . \text { concat }(r, a(t, d)(v, r))\end{aligned}$

meaning

$\lambda k: K_{1} \cdot a^{\prime}\left(T_{a}(k)\right) \circ a(k)$

$\lambda k: K_{1} \cdot \lambda v: k \cdot\left(a(k)(v), a^{\prime}(k)(v)\right)$

$\lambda k: K_{1}, \lambda v: k \cdot u n i o n\left(a(k)(v), a^{\prime}(k)(v)\right)$

$\lambda d: D . \lambda r: d . \operatorname{concat}(r, a(d)(r))$

where:

Figure 6

D: Declaration $\rightarrow(D \rightarrow D)$

$D \llbracket D_{1}$ and $D_{2} \rrbracket=D \llbracket D_{1} \rrbracket * D \llbracket D_{2} \rrbracket$

$\mathrm{D} \llbracket \mathrm{I}=\mathrm{E} \rrbracket=\mathrm{E} \llbracket \mathrm{E} \rrbracket ;$ bind $\mathrm{I}$

E: Expression $\rightarrow(D \rightarrow F)$

$\mathrm{E}[\mathrm{D}$ in $\mathrm{E} \rrbracket=(\triangleright \mathrm{D} \llbracket \mathrm{D} \rrbracket) ; \mathrm{E} \llbracket \mathrm{E} \rrbracket$

$\mathrm{E}[\mathrm{N}: t \rrbracket=p u t(\mathrm{~N}: t)$

$E\left[\mid o p E_{1} E_{2} \rrbracket=\left(E\left[E_{1} \rrbracket * E\left[E_{2} \rrbracket\right) ; o p\right.\right.\right.$

E[II] = find I

E[lambda I: $t, \mathrm{E}]=$ freeze $_{i}\left(\left(\triangleright\left(\right.\right.\right.$ forget $_{D} ;$ bind $\left.\left.\mathrm{I}\right)\right) ; \mathrm{E}[\mathrm{E} \rrbracket)$

$\mathrm{E}_{[}\left[\mathrm{E}_{1} \mathrm{E}_{2} \rrbracket=\left(\mathbf{E}\left[\mathrm{E}_{1} \rrbracket * \mathrm{E}_{[}\left[\mathrm{E}_{2} \rrbracket\right) ;\right.\right.\right.$ eval

$a_{0}=(\triangleright(p u t(2:$ int $) ;$ bind $B)) ;($ find $A *$ find $B) ;$ add

in the sense that every typing $d_{0} \rightarrow t_{0}$ that results from a ground substitution into the typing scheme conforms with the typing function: $T_{a_{0}}\left(d_{0}\right)=t_{0}$. Also, for any type $d_{0} \neq$ ns such that $T_{a_{0}}\left(d_{0}\right)=t_{0} \neq \mathrm{ns}$, there exists a ground substitution into the scheme that yields the typing $d_{0} \rightarrow t_{0}$. If such properties hold, we say that the typing scheme is sound and complete, respectively. In [5], we showed the proof of soundness and completeness of a method of type inference for action semantics expressions. In this section, we give the algorithms that implement the method. 
Polymorphic type inference originated with ML [9] and was extended for subtypes by Mitchell [10]; implementations were undertaken by Fuh and Mishra [6,7]. Subtyping was represented by a set of constraints of the form $\tau_{1} \leq \tau_{2}$, where $\tau_{1}$ and $\tau_{2}$ represent typing schemes. Problems arose: subtyping constraints crept in at every function application, so even small programs had typing schemes whose constraint sets were unmanageable [7]. Also, checking satisfiability of constraints was not easy [6,16]. Cardelli [1], Cardelli and Wegner [3], Cardelli and Mitchell [2], Stansifer [22], Wand [24], Jategaonker and Mitchell [8], and Remy [17] studied subtyping of record types. In particular, Wand [24] noted the need for a complex constraint on the typing scheme for the record concatenation operation.

The type inference algorithm in this paper handles subtypes, records, and record concatenation and union operations yet avoids large constraint sets and constraints on records. Since action semantics is built on a model where actions are natural transformations, few constraints are needed to obtain soundness and completeness properties for type inference. Since the action semantics notation is more constrained than that studied by Wand, constraints on record concatenation and union are avoided. Thus, we achieve the goal of simple, ML-style type inference for a notation with subtypes and records.

We now give the syntax of typing schemes for actions. A typing scheme has the form:

$$
S_{1} \rightarrow S_{2} \text { if } C
$$

where:

SETyping-scheme
CEConstraint
$v \in$ Functional-facet-scheme
d $\in$ Declarative-facet-scheme
$f \in$ Field-scheme
$S::=v \mid d$
$v::=$ int $\mid$ real $|\cdots| v_{1} \times v_{2}\left|v_{1} \rightarrow v_{2}\right| \theta$
$d::=\left\{i: f_{i}\right\}_{i \in I} \alpha\left|\left\{i: f_{i}\right\}_{i \in I \text { exactly }}\right|\left\{i: f_{i}\right\}_{i \in I} \alpha_{\text {exactly }}$
$f::=$ absent | present(v) | $\Delta$
$C::=v_{1} \leq v_{2} \mid C_{1}$ and $C_{2} \mid$ true

Placeholders ("variables") are represented by $\theta, \Delta$, and $\alpha . \theta$ is a variable for an arbitrary proper type in the functional facet. $\Delta$ is a field variable $[17,24]$; the phrase $i: \Delta$ represents unknown status about the presence or absence of identifier $i$ (and its type) in a record type. $\alpha$ is a row variable [17,24]; the scheme $\left\{i: f_{i}\right\}_{i \in I} \alpha$ represents unknown status about all identifiers $j \notin I$ in the record type. For example, the scheme $\{A: p r e s e n t(\theta) ; B: \Delta ; C: a b s e n t\} \alpha_{\text {exacty }}$ represents the family of record types that have an A-field, might (or might not) have a $\mathrm{B}$-field, definitely do not have a $\mathrm{C}$-field, might (or might not) have other fields beyond $A, B$, and $C$, and finally, have no hidden fields. Field and row variables are used for "bookkeeping" during type inference; their use leads to simple definitions of unification and inference for record operations. As in Tofte [23], Remy [17], and Wand [24], we assume that a row variable $\alpha$, as appearing in $\left\{i: f_{i}\right\}_{i \in l} \alpha$, has as its "domain of use" $I$. That is, $\alpha$ can be instantiated only with fields whose labels are not in $I$.

We use the usual notion of substitution [21] and use $U$ to stand for an arbitrary substitution. A specific substitution is written $\left[j \mapsto S_{j}\right]_{j \in J}$. The function unify:Typing-schemexTypingscheme $\rightarrow$ Substitution does first-order unification [21].

Figure 7 gives the type schemes for the actions in Figure 3, and Figure 8 gives the typing schemes for the compositions. Type inference for sequential composition depends on the unification of the target scheme for action $a$ and the source scheme of action $a^{\prime}$. (Note that the constraints of the two action's schemes must be satisfied, else the composition defines a "nonsense" mapping.) MLstyle unification is used for unification of nonrecord typing schemes. When record schemes are unified, a simplified version of Tofte's unification algorithm is employed [23]. Its definition is given in Figure 9. (For example, the unification of $\left\{A: \Delta_{1} ; C: a b s e n t\right\} \alpha$ to 
Figure 7

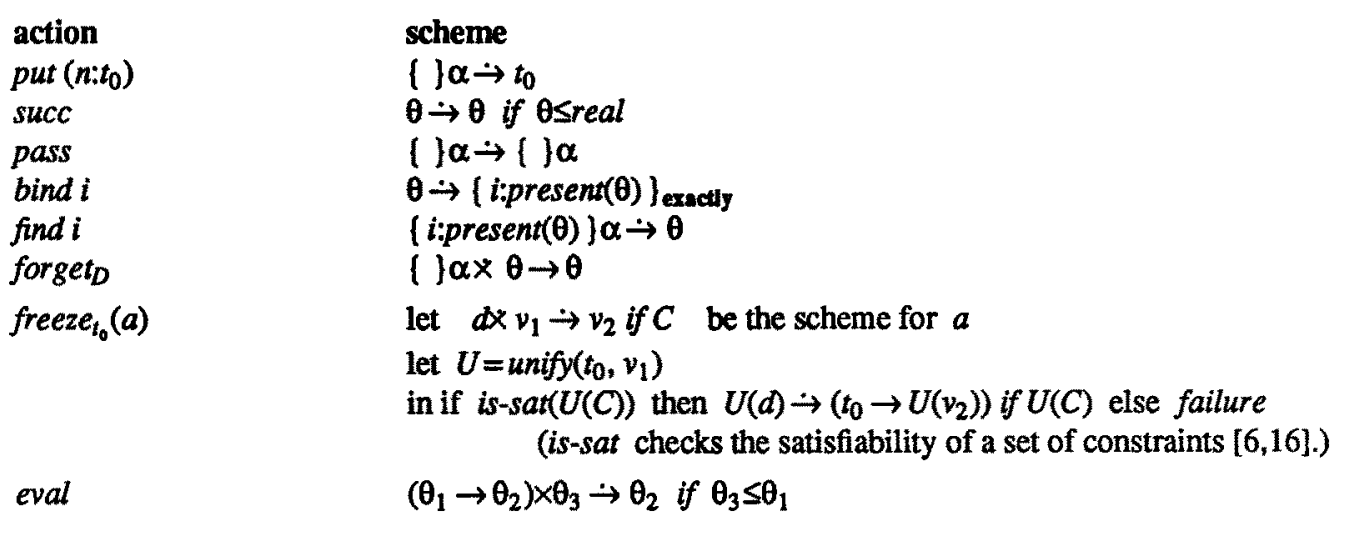

Figure 8

let the scheme for action $a$ be $S_{1} \rightarrow S_{2}$ if $C$

and the scheme for action $a^{\prime}$ be $S_{1} \rightarrow S_{2}{ }^{\prime}$ if $C^{\prime}$

action typing scheme

$a ; a^{\prime} \quad$ if $U=$ unify $\left(S_{2}, S_{1}\right) \neq$ failure

and is-sat $(U(C$ and $C))$

then $U\left(S_{1}\right) \rightarrow U\left(S_{2}\right)$ if $U(C$ and $C)$

else failure

$a * a^{\prime} \quad$ if $U=$ unify $\left(S_{1}, S_{1}\right) \neq$ failure

and $U^{\prime}, S^{\prime}=S_{\text {marge }}\left(U\left(S_{2}\right), U\left(S_{2}\right)\right) \neq$ failure

and is-sat $\left(U^{\circ} \circ U(C\right.$ and $\left.C)\right)$

then $U^{\prime} \circ U\left(S_{1}\right) \rightarrow S^{\prime}$ if $U^{\prime} \circ U\left(C\right.$ and $\left.C^{\prime}\right)$

else failure

$\triangleright a \quad$ if $U, S=S_{\text {concat }}$ (declarative-facet-scheme-in $\left.\left(S_{1}\right), S_{2}\right) \neq$ failure

and is-sat $(U(C))$

then $U\left(S_{1}\right) \rightarrow S$ if $U(C)$

else failure 
Figure 9

unify-record: Declarative-facet-scheme $\times$ Declarative-facet-scheme $\rightarrow$ Substitution

unify-record $\left(d_{1}, d_{2}\right)=$

let $I=$ fields-in $\left(d_{1}\right) \cup$ fields-in $\left(d_{2}\right)$

let $U_{1}, d_{1}{ }^{\prime}=$ extend-record-with-new-fields $\left(N\right.$ fields-in $\left.\left(d_{1}\right), d_{1}\right)$

let $U_{2}, d_{2}{ }^{\prime}=$ extend-record-with-new-fields $\left(I\right.$ fields-in $\left.\left(d_{2}\right), d_{2}\right)$

let $\left\{\right.$ fields $\left._{1}\right\}$ row $_{1}=d_{1}{ }^{\circ}$

let $\left\{\right.$ fields $\left._{2}\right\}$ row $_{2}=d_{2}$ '

let $U_{3}=$ unify-row-variables $\left(\right.$ row $_{1}$, row $\left._{2}\right)$

let $U_{4}=$ unify-fields $\left(\right.$ field $s_{1}$, fields $s_{2}$ )

in $U_{4} \circ U_{3} \circ U_{2} \circ U_{1}$

where:

extend-record-with-new-fields $\left(J,\left\{i: f_{i}\right\}_{i \in I \text { exactly }}\right)=[],\left\{i: f_{i}\right\}_{i \in I} @\{j: a b s e n t\}_{j \in J \text { exactly }}$ extend-record-with-new-fields $\left(J,\left\{i: f_{i}\right\}_{i \in I} \alpha\right)=\left[\alpha \mapsto\left\{j: \Delta_{j}\right\}_{j \in J} \gamma\right],\left\{i: f_{i}\right\}_{i \in I} @\left\{j: \Delta_{j}\right\}_{j \in J} \gamma$ extend-record-with-new-fields $\left(J,\left\{i: f_{i}\right\}_{i \in I} \alpha_{\text {exactly }}\right)=\left[\alpha \mapsto\left\{j: \Delta_{j}\right\}_{j \in J} \gamma\right],\left\{i: f_{i}\right\}_{i \in I} @\left\{j: \Delta_{j}\right\}_{j \in J} \gamma_{\text {exactly }}$

(assume variables $\Delta_{j}$ and $\gamma$ are newly generated)

unify-row-variables $($ exactly, exactly $)=[]$

unify-row-variables $(\alpha$, exactly $)=\left[\alpha \mapsto \rightarrow(\}_{\text {exactly }}\right]$

unify-row-variables $(\alpha, \beta)=[\alpha \mapsto \gamma][\beta \mapsto \gamma]$

unify-row-variables $\left(\alpha, \beta_{\text {exactly }}\right)=\left[\alpha \mapsto \gamma_{\text {exacty }}\right][\beta \mapsto \gamma]$

unify-row-variables $(\alpha$ exactly, exactly $)=[\alpha \mapsto \mid\}]$

unify-row-variables $\left(\alpha_{\text {exactly }}, \beta_{\text {exactly }}\right)=[\alpha \mapsto \gamma][\beta \mapsto \gamma]$

(assume variable $\gamma$ is newly generated)

(the operation is commutative)

unify-fields $\left(d_{1}, d_{2}\right)=$

if $d_{1}=d_{2}=\{\}$ then []

else let $\left\{i: f_{1}\right\} @ d_{1}^{\prime}=d_{1}$

let $\left\{i: f_{2}\right\} @ d_{2}{ }^{\prime}=d_{2}$

let $U_{1}=$ unify $\left(f_{1}, f_{2}\right)$

let $U_{2}=$ unify-fields $\left(U_{1}\left(d_{1}\right), U_{1}\left(d_{2}\right)\right)$

in $U_{2} \circ U_{1}$

$\left\{A \text { :present }\left(\theta_{2}\right): B \text { :present(bool) }\right\}_{\text {exacty }}$ is the substitution: $\left[\alpha \mapsto\left\{B: \Delta_{2}\right\} \beta\right] \quad\left[\beta \mapsto\{\}_{\text {exactly }}\right]$ $\left[\Delta_{1} \mapsto \operatorname{present}\left(\theta_{2}\right)\right]\left[\Delta_{2} \mapsto \operatorname{present}(\mathrm{bool})\right]$, which produces the scheme:

$\left\{A \text { :present }\left(\theta_{2}\right) ; B \text { :present(bool); } C \text { :absent }\right\}_{\text {exactly }}$, which is equivalent to:

$\left\{\text { A:present }\left(\theta_{2}\right) ; B \text { :present }(\text { bool })\right\}_{\text {exactly }}$.

Parallel composition depends on the inference defined by $S_{\text {merge }}$. See Figure 10 . When merging functional facet values, ordinary tupling occurs, so a product type is inferred. When merging declarative records, the records are unioned if they have no fields in common. The type inference must verify that the types of the two records have no fields in common and both record type schemes are "exactly." The key to keeping the inference simple is the omission of some of the cases in functions resolve-row-variables and resolve-fields. The omitted cases create complex record constraints for unioning typing schemes like $\{\cdots\} \alpha$ to $\{\cdots\} \beta$ or unioning $\left\{A: \Delta_{1}\right\}_{\text {exactly }}$ to $\left\{A: \Delta_{2}\right\}_{\text {exactly }}[24]$. We will show that the typing schemes for action expressions have properties that make the omitted cases impossible in our system. 
$S_{\text {merge }}:$ Typing-scheme $\times$ Typing-scheme $\rightarrow$ Substitution $\times$ Typing-scheme

$S_{\text {merge }}\left(v_{1}, v_{2}\right)=[], v_{1} \times v_{2}$

$S_{\text {merge }}\left(d_{1}, d_{2}\right)=$

let $I=$ fields-in $\left(d_{1}\right) \cup$ fields-in $\left(d_{2}\right)$

let $U_{1}, d_{1}{ }^{\prime}=$ extend-record-with-new-fields $\left(\Lambda\right.$ fields-in $\left.\left(d_{1}\right), d_{1}\right)$

let $U_{2}, d_{2}{ }^{\prime}=$ extend-record-with-new-fields $\left(N\right.$ fields-in $\left.\left(d_{2}\right), d_{2}\right)$

let $U_{3}, d=S_{\text {ynion }}\left(d_{1} ; d_{2}\right)$

in $U_{3} \circ U_{2} \circ U_{1}, d$

where:

$S_{\text {union }}\left(\left\{\right.\right.$ fields $\left._{1}\right]$ rrow $_{1},\left[\right.$ fields $\left._{2}\right]$ row $\left._{2}\right)=$

let $U_{1}$, row $=$ resolve-row-vars $\left(\right.$ row $_{1}$, row $\left._{2}\right)$

let $U_{2}$, fields $=$ resolve-fields $\left(f i e l d s_{1}\right.$, field $_{2}$ )

in $U_{2} \circ U_{1},[$ fields $]$ row

where:

resolve-row-vars (exactly, exactly) $=$ [], exactly

resolve-row-vars $\left(\alpha_{\text {exactly, exactly }}\right)=[], \alpha_{\text {exactly }}$

resolve-row-vars $(\alpha$, exactly $)=\left[\alpha \rightarrow \beta_{\text {exacty }}\right], \beta_{\text {exactly }}$

resolve-row-vars $(\alpha, \alpha)=\left[\alpha \mapsto\{\}_{\text {exacty }}\right]$, exactly

resolve-row-vars $\left(\alpha_{\text {exactly }}, \alpha_{\text {exactly }}\right)=[\alpha \rightarrow[1]$, exactly

resolve-row-vars $(\alpha[$ exactly $], \beta[$ exactiy $])=$ do not appear in our system

(the italic brackets enclose optional phrases)

(the operation is commutative)

resolve-fields $\left(d_{1}, d_{2}\right)=$

if $d_{1}=d_{2}=\{\}$ then [], \{ \}

else let $\left\{i: f_{1}\right\} @ d_{1}^{\prime}=d_{1}$

let $\left\{i: f_{2}\right\} @ d_{2}=d_{2}$

let $U_{1}, f^{\prime}=\operatorname{cases}\left(f_{1}, f_{2}\right)$ of

(absent, $f$ ) or $(f$, absent $):[], f$

$(\Delta, \Delta):[\Delta \mapsto a b s e n t]$, absent

$(\Delta$, present $(v))$ or $($ present $(v), \Delta):[\Delta \mapsto a b s e n t]$, present $(v)$

(present( $v)$, present $(v))$ : failure

$(\Delta, \Delta)$ : does not appear in our system

let $U_{2}, d=$ resolve-fields $\left(U_{1}\left(d_{1}\right), U_{1}\left(d_{2}\right)\right)$

in $U_{2} \circ U_{1},\left\{i: f^{\prime}\right\} @ d$

Concatenation composition depends on the inference defined by $S_{\text {concal }}$. See Figure 11 . $S_{\text {concat }}\left(d_{1}, d_{2}\right)$ requires that $d_{2}$ has an "exactly" typing (cf. Figure 5). Problems arise when row and field variables appear in $d_{2}$, because unknown bindings are overriding existing ones. Fortunately, action semantics expressions have the property that any row and field variables in $d_{2}$ 's typing scheme must be present in $d_{1}$ 's, which represents the harmless case where an unknown binding is overriding. itself. The omitted cases in Figure 11 never arise.

Despite the omitted cases, the definitions in Figures 10 and 11 are well-defined for the restricted actions we use. There are two crucial properties: a scheme $S \rightarrow T$ if $C$ has the $\alpha$-preserved property 
Figure 11

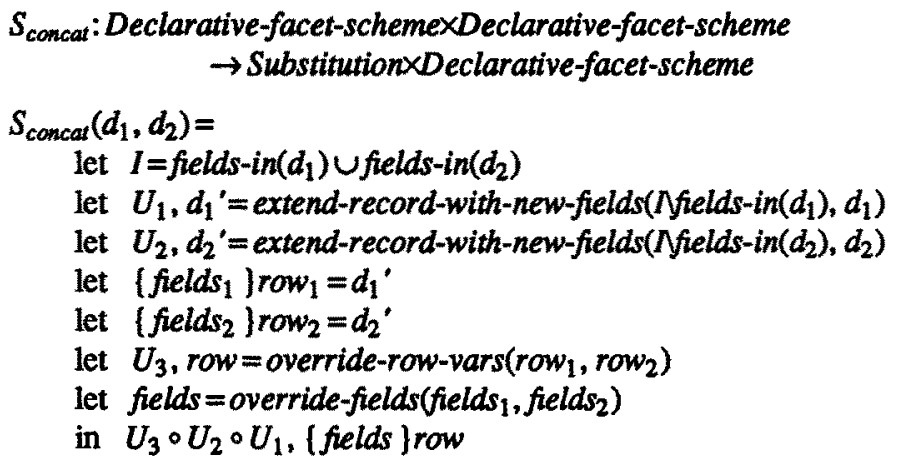

where

override-row-vars $\left(\right.$ row $_{1}$, exactly $)=[]$, row $_{1}$

override-row-vars $\left(\alpha_{\text {exactly }}, \alpha_{\text {exactly }}\right)=[], \alpha_{\text {exactly }}$

override-row-vars $(\alpha, \alpha)=\left[\alpha \mapsto \beta_{\text {exacty }}\right], \beta_{\text {exactly }}$

override-row-vars(all other cases) $=$ do not appear in our system

override-fields $\left(d_{1}, d_{2}\right)=$

if $d_{1}=d_{2}=\{\}$ then \{\}

else let $\left\{i: f_{1}\right\} @ d_{1}{ }^{\prime}=d_{1}$

let $\left[i: f_{2}\right\} @ d_{2}{ }^{\prime}=d_{2}$

let $f^{\prime}=\operatorname{cases}\left(f_{1}, f_{2}\right)$ of

$(f, a b s e n t): f$

$(f, p r e s e n t(v)): p r e s e n t(v)$

$(\Delta, \Delta): \Delta$

$(\Delta, \Delta)$ or (absent, $\Delta)$ or (present $(v), \Delta)$ : do not appear in our system

let $d=$ override-fields $\left(d_{1}, d_{2}\right)$

in $\left\{i: f^{\prime}\right\} @ d$

if, whenever $T$ is a declarative facet scheme containing a row variable $\alpha$, then $S$ is a declarative facet scheme containing the same $\alpha$, and it has the $\Delta$-preserved property if, whenever $T$ is a declarative facet scheme containing a record field $i: \Delta$, then $S$ is a declarative facet scheme containing the same $i: \Delta$.

Proposition: Let $a_{i}: S_{i} \rightarrow S_{i}^{\prime}$ if $C_{i}, \quad i \in 1.2$; let $U=$ unify $\left(S_{1}, S_{2}\right) \neq$ failure; let $U\left(a_{i}\right)=U\left(S_{i}\right) \rightarrow U\left(S_{i}\right)$ if $U\left(C_{i}\right)$. If $a_{1}$ and $a_{2}$ have the $\alpha$-preserved and $\Delta$-preserved properties for all row variables $\alpha$ and field variables $\Delta$, then so have $U\left(a_{1}\right)$ and $U\left(a_{2}\right)$.

Proof: All row and field variables are created by substitution into row variables. The result follows from the $\alpha$-preserved property.

Consider the inference for $a_{1} * a_{2}$, where $a_{i}: d_{i} \rightarrow d_{i}^{\prime}$ if $C_{i}, i \in 1.2$. Assume that the schemes have the $\alpha$ - and $\Delta$-preserved properties. The type inference unifies $d_{1}$ with $d_{2}$ (giving substitution $U)$, forcing the row and field variables to be the same in $U\left(d_{1}\right)$ and $U\left(d_{2}\right)$. If $U\left(d_{i}\right)$ has a row variable, by the Proposition, it is in $U\left(d_{i}\right)$ as well. So, if both $U\left(d_{1}\right)$ and $U\left(d_{2}\right)$ have row variables, 
they must be identical, implying that the two arguments to $S_{\text {union }}$ have the same row variables- the omitted cases in Figure 10 never arise. A similar result holds for field variables in $U\left(d_{1}\right)$ and $U\left(d_{2}\right)$.

For inference on $>a$, if $a^{\prime}$ s scheme $d \rightarrow d^{\prime}$ if $C$ has the $\alpha$ - and $\Delta$-preserved properties, then the row and field variables in $d^{\prime}$ will be present in $d$. The omitted cases in Figure 11 never arise.

We now consider soundness and completeness. Say that a substitution into a typing scheme is a ground substitution if no variables remain after the substitution is applied to the scheme. A ground substitution maps a typing-scheme into a type. An instantiated scheme such as \{ A:absent; B:present(bool) \}exactly is read as the type [B:bool \}exactly, that is, "absent" fields are dropped when the record is "exactly." When a ground substitution yields an instantiated scheme like \{A:absent \}, that is, an "absent" field in a "non-exactly" record, we disallow the substitution, since the instantiated scheme has no interpretation in our typing system. (But such a type is legal in [2].)

Definition: A typing-scheme $S_{1} \rightarrow S_{2}$ if $C$ is sound for action $a: K_{1} \rightarrow K_{2}$ iff for all $k \in K_{1}$, for all ground substitutions $U, U\left(S_{1}\right)=k \neq \mathrm{ns}$, and $U(C)$ holds true imply $T_{a}(k)=U\left(S_{2}\right)$.

The typing-scheme is complete for action $a$ iff for all $k \in K_{1}, T_{a}(k) \neq \mathbf{n s}$ implies there exists a ground substitution $U$ such that $U\left(S_{1}\right)=k$ and $U(C)$ holds true.

In an earlier paper [5], we showed that the typing schemes in Figure 7 and the type inference in Figure 8 give sound and complete typing schemes for action semantics expressions. A key to the results is that the typing functions $T_{a}$ are monotonic with respect to the subtyping ordering. Thus, the domain of a typing function is a down-closed set in the underlying partial ordering of types.

This structural property of domains of typing functions gives an advantage for type inference: it reduces the size of constraint sets, because the typing schemes naturally represent down-closed sets. Further, composition of actions introduces no new constraints into the constraints set of the typing scheme for the composed action, since the typing functions (which describe the behavior of natural transformations) must compose. Constraints appear only when the domain of an action must be further restricted (e.g., succ). In contrast, in the Mitchell/Fuh\&Mishra system $[6,7,8,10]$ constraints represent coercion functions. For example, the identity mapping has the scheme: $\theta_{1} \rightarrow \theta_{2}$ if $\theta_{1} \leq \theta_{2}$, and the composition of $a: \theta_{1} \rightarrow \theta_{2}$ with $a^{\prime}: \theta_{1}{ }^{\prime} \rightarrow \theta_{2}{ }^{\prime}$ generates the constraint $\theta_{2} \leq \theta_{1}$ :

\section{An Example}

We now give an example of type inference. Consider this program in the language in Figure 6:

$$
F=(\text { lambda } C \text { :real. } \operatorname{div} C B \text { ) and } A=3 \text { :int in } F A
$$

The action semantics denotation of the program is $a_{7}$, where:

$$
\begin{aligned}
& a_{7}=\left(\triangleright a_{5}\right) ; a_{6} \\
& a_{6}=\mathrm{E} \llbracket F A \rrbracket=(\text { find } F * \text { find } A) ; \text { eval } \\
& a_{5}=\mathrm{D} \llbracket F=(\operatorname{lambda} C \text { :real. } \operatorname{div} C B) \text { and } A=3: \operatorname{int} \rrbracket=a_{4} * a_{1} \\
& a_{4}=\mathrm{D} \llbracket F=\operatorname{lambda} C \text { :real. } \operatorname{div} C B \rrbracket=a_{3} ; \text { bind } F \\
& a_{3}=\mathrm{E} \llbracket \text { lambda } C \text { :real. } \operatorname{div} C B \rrbracket=\text { freeze } e_{\text {real }}\left(\left(>\left(\text { forget }_{D} ; \text { bind } C\right)\right) ; a_{2}\right) \\
& a_{2}=\mathrm{E} \llbracket \operatorname{div} C B \rrbracket=(\text { find } C * \text { find } B) ; \operatorname{div} \\
& a_{1}=\mathrm{D} \llbracket A=3: \operatorname{int} \rrbracket=\text { put }(3 \text { :int }) ; \text { bind } A
\end{aligned}
$$

We will write $a \in S_{1} \rightarrow S_{2}$ if $C$ to denote that $a$ has the indicated typing scheme.

Type inference for $a_{1}$ :

$$
\text { put(3:int) } \in\{\} \alpha_{1} \rightarrow \text { int }
$$

bind $A \in \theta_{1} \rightarrow\left\{A \text { :present }\left(\theta_{1}\right)\right\}_{\text {exactly }}$ 
(put(3:int); bind $A) \in\{\} \alpha_{1} \rightarrow\{A \text { :present(int) }\}_{\text {exactly }}$ and $U=\left[\theta_{1} \mapsto\right.$ int $]$

Type inference for $a_{2}$ :

find $C \in\left\{C: p r e s e n t\left(\theta_{2}\right)\right\} \alpha_{2} \rightarrow \theta_{2}$

find $B \in\left\{B\right.$ :present $\left.\left(\theta_{3}\right)\right\} \alpha_{3} \rightarrow \theta_{3}$

(find $C *$ find $B) \in\left\{C:\right.$ present $\left.\left(\theta_{2}\right), B: p r e s e n t\left(\theta_{3}\right)\right\} \alpha_{4} \rightarrow \theta_{2} \times \theta_{3}$

and $U=\left[\alpha_{2} \mapsto\left\{B: p r e s e n t\left(\theta_{3}\right)\right] \alpha_{4}\right]\left[\alpha_{3} \mapsto\left\{C: p r e s e n t\left(\theta_{2}\right)\right\} \alpha_{4}\right]$

div $\in \theta_{4} \times \theta_{5} \rightarrow$ real if $\theta_{4} \leq$ real and $\theta_{5} \leq$ real

(find $C *$ find $B) ;$ div $\in\left\{C\right.$ :present $\left(\theta_{6}\right), B:$ present $\left.\left(\theta_{7}\right)\right\} \alpha_{4} \rightarrow$ real if $\theta_{6} \leq$ real and $\theta_{7} \leq$ real and $U=\left[\theta_{4} \mapsto \theta_{6}\right]\left[\theta_{2} \mapsto \theta_{6}\right]\left[\theta_{5} \mapsto \theta_{7}\right]\left[\theta_{3} \mapsto \theta_{7}\right]$

Type inference for $a_{3}$ :

$$
\begin{aligned}
& \text { forget }_{D} \in\{\} \alpha_{5} \times \theta_{8} \rightarrow \theta_{8} \\
& \text { bind } C \in \theta_{9} \rightarrow\left\{C \text { :present }\left(\theta_{9}\right)\right\}_{\text {exactly }}
\end{aligned}
$$

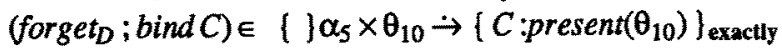

$$
\begin{aligned}
& \text { and } U=\left[\theta_{8} \mapsto \theta_{10}\right]\left[\theta_{9} \mapsto \theta_{10}\right] \\
& >\left(\text { forget }_{D} ; \text { bind } C\right) \in\left\{C: \Delta_{1}\right\} \alpha_{6} \times \theta_{10} \rightarrow\left\{C: \operatorname{present}\left(\theta_{10}\right)\right\} \alpha_{6} \\
& \text { and } U=\left[\alpha_{5} \mapsto\left\{C: \Delta_{1}\right\} \alpha_{6}\right] \\
& a_{2} \in\left\{C \text { :present }\left(\theta_{6}\right), B \text { :present }\left(\theta_{7}\right)\right\} \alpha_{4} \rightarrow \text { real if } \theta_{6} \leq \text { real and } \theta_{7} \leq \text { real } \\
& \left(p\left(\text { forget }_{D} ; \text { bind } C\right)\right) ; a_{2} \in\left\{B \text { :present }\left(\theta_{7}\right), C: \Delta_{1}\right\} \alpha_{7} \times \theta_{11} \rightarrow \text { real if } \theta_{11} \leq \text { real and } \theta_{7} \leq \text { real } \\
& \left(\text { freeze }_{\text {real }}\left(\left(\triangleright\left(\text { forget }_{D} ; \text { bind } C\right)\right) ; a_{2}\right)\right) \in \\
& \left.\left\{B: p r e s e n t\left(\theta_{7}\right), C: \Delta_{1}\right\} \alpha_{7} \rightarrow \text { (real } \rightarrow \text { real }\right) \text { if } \theta_{7} \leq \text { real } \\
& \text { and } U=\left[\theta_{11} \mapsto \text { real }\right] \\
& U\left(\theta_{11} \leq \text { real }\right)=\text { real } \leq \text { real }=\text { true }
\end{aligned}
$$$$
\text { and } U=\left[\theta_{10} \mapsto \theta_{11}\right]\left[\theta_{6} \mapsto \theta_{11}\right]\left[\alpha_{6} \mapsto\left[B: p r e s e n t\left(\theta_{7}\right)\right] \alpha_{7}\right]\left[\alpha_{4} \mapsto \alpha_{7}\right]
$$

Type inference for $a_{4}$ :

$a_{3} \in\left\{B:\right.$ present $\left.\left(\theta_{7}\right), C: \Delta_{1}\right\} \alpha_{7} \rightarrow$ (real $\rightarrow$ real) if $\theta_{7} \leq$ real

bind $F \in \theta_{12} \rightarrow\left\{F: \text { present }\left(\theta_{12}\right)\right\}_{\text {exactly }}$

$a_{3} ;$ bind $F \in\left\{B: p r e s e n t\left(\theta_{7}\right), C: \Delta_{1}\right\} \alpha_{7} \rightarrow\{F$ present(real $\rightarrow$ real $\left.)\right\}_{\text {exactly }}$ if $\theta_{7} \leq$ real and $U=\left[\theta_{12} \mapsto\right.$ (real $\rightarrow$ real $\left.)\right]$

Type inference for $a_{5}$ :

$$
\begin{aligned}
& a_{1} \in\{\} \alpha_{1} \rightarrow\{A: \text { present(int) }\}_{\text {exactly }} \\
& \left.a_{4} \in\left\{B: p r e s e n t\left(\theta_{7}\right), C: \Delta_{1}\right\} \alpha_{7} \rightarrow\{F: \text { present(real } \rightarrow \text { real })\right\}_{\text {exactly }} \text { if } \theta_{7} \leq \text { real } \\
& a_{4} * a_{1} \in\left\{B: \text { present }\left(\theta_{7}\right), C: \Delta_{1}\right\} \alpha_{8} \rightarrow\{A: \text { present(int), } F: \text { present(real } \rightarrow \text { real) }\}_{\text {exactly }} \\
& \quad \text { if } \theta_{7} \leq \text { real } \\
& \quad \text { and } U=\left[\alpha_{1} \mapsto\left\{B: p r e s e n t\left(\theta_{7}\right), C: \Delta_{1}\right\} \alpha_{8}\right]\left[\alpha_{7} \mapsto \alpha_{8}\right\}
\end{aligned}
$$

Type inference for $a_{6}$ :

$$
\begin{aligned}
& \text { find } F \in\left\{F: \text { present }\left(\theta_{13}\right)\right\} \alpha_{9} \rightarrow \theta_{13} \\
& \text { find } A \in\left\{A: p r e s e n t\left(\theta_{14}\right)\right\} \alpha_{10} \rightarrow \theta_{14} \\
& \text { find } F * \text { find } A \in\left\{F: p r e s e n t\left(\theta_{13}\right), A: p r e s e n t\left(\theta_{14}\right)\right\} \alpha_{11} \rightarrow \theta_{13} \times \theta_{14} \\
& \quad \text { and } U=\left[\alpha_{9} \mapsto\left\{A: p r e s e n t\left(\theta_{14}\right)\right\} \alpha_{11}\right]\left[\alpha_{10} \mapsto\left\{F: p r e s e n t\left(\theta_{13}\right)\right\} \alpha_{11}\right] \\
& \text { eval } \in\left(\theta_{15} \rightarrow \theta_{16}\right) \times \theta_{17} \rightarrow \theta_{16} \text { if } \theta_{17} \leq \theta_{15}
\end{aligned}
$$


(find $F *$ find $A)$; eval $\in\left\{F\right.$ :present $\left(\theta_{15} \rightarrow \theta_{16}\right), A$ :present $\left.\left(\theta_{18}\right)\right\} \alpha_{11} \rightarrow \theta_{16}$ if $\theta_{18} \leq \theta_{15}$ and $U=\left[\theta_{13} \mapsto\left(\theta_{15} \rightarrow \theta_{16}\right)\right]\left[\theta_{14} \mapsto \theta_{18}\right]\left[\theta_{17} \mapsto \theta_{18}\right]$

Type inference for $a_{7}$ :

$$
\begin{aligned}
& a_{5} \in\left\{B: p r e s e n t\left(\theta_{7}\right), C: \Delta_{1}\right\} \alpha_{8} \rightarrow\{A: \text { present(int), } F: \text { present }(\text { real } \rightarrow \text { real })\}_{\text {exactly }} \text { if } \theta_{7} \leq \text { real } \\
& >a_{5} \in\left\{B: \text { present }\left(\theta_{7}\right), C: \Delta_{1}, A: \Delta_{2}, F: \Delta_{3}\right\} \alpha_{12} \\
& \left.\left.\rightarrow\left\{B: p r e s e n t\left(\theta_{7}\right), A: \text { present(int }\right), F: \text { present(real } \rightarrow \text { real }\right), C: \Delta_{1}\right\} \alpha_{12} \\
& \quad \text { if } \theta_{7} \leq \text { real } \\
& \quad \text { and } U=\left[\alpha_{8} \mapsto\left\{A: \Delta_{2}, F: \Delta_{3}\right\} \alpha_{12}\right] \\
& a_{6} \in\left\{F: p r e s e n t\left(\theta_{15} \rightarrow \theta_{16}\right), A: p r e s e n t\left(\theta_{18}\right)\right\} \alpha_{11} \rightarrow \theta_{16} \text { if } \theta_{18} \leq \theta_{15} \\
& \left(\triangleright a_{5}\right): a_{6} \in\left\{B: p r e s e n t\left(\theta_{7}\right), C: \Delta_{1}, A: \Delta_{2}, F: \Delta_{3}\right\} \alpha_{13} \rightarrow \text { real if } \theta_{7} \leq \text { real } \\
& \quad \text { and } U=\left[\alpha_{11} \mapsto\left\{B: p r e s e n t\left(\theta_{7}\right), C: \Delta_{1}\right\} \alpha_{13}\right]\left[\theta_{15} \mapsto \text { real }\right]\left[\theta_{16} \mapsto \text { real }\right]\left[\theta_{18} \mapsto \text { int }\right]\left[\alpha_{12} \mapsto \alpha_{13}\right] \\
& \quad U\left(\theta_{18} \leq \theta_{15}\right)=\text { int } \leq \text { real }=\text { true }
\end{aligned}
$$

So, the typing scheme is:

$$
\left\{B \text { :present }\left(\theta_{7}\right), C: \Delta_{1}, A: \Delta_{2}, F: \Delta_{3}\right\} \alpha_{13} \rightarrow \text { real if } \theta_{7} \text { sreal }
$$

The field variables in the scheme's source are just "little row variables;" since neither they nor the row variable $\alpha_{13}$ appear in the scheme's target or constraints, we compress them into:

$$
\left\{B \text { :present }\left(\theta_{7}\right)\right\} \alpha^{\prime} \rightarrow \text { real if } \theta_{7} \leq \text { real }
$$

The compression of field variables with row variables can (and should) also be undertaken in the intermediate steps above, but we chose not to do so, in order that the inference steps matched the definitions in Figures 7-11.

The scheme for the program tells us that the program requires a declarative record argument that has a binding of $B$ to a value with a subtype of real so that the program can produce a real-typed answer. By the soundness and completeness properties, the scheme describes all the well-defined behaviors of the program. Thus, the scheme is useful for program analysis and code improvement.

\section{Conclusion}

We have shown how to perform type inference for action semantics expressions in a fashion that avoids large constraint sets and constraints on record types for record union and concatenation operations. We have implemented the type inference algorithm as well as an interpreter for action semantics [4].

Although constraints on record types are unnecessary for record union and concatenation, they appear in the typing schemes for the freeze and eval actions for the declarative facet. (The actions are used to model definition and invocation of statically scoped procedures.) We have extended the type inference algorithm in this paper to handle constraints on records.

The subset of action semantics presented in this paper is appropriate for statically typed languages. Of course, full action semantics can give semantics to dynamically typed languages as well. We model dynamic typing with an additional type called dynamic and the subtyping constraint $t \leq$ dynamic for all proper functional types $t$. Type inference with dynamic types has a problem: the "occurs check" [6], which is used to detect that a constraint like $\theta \times$ int $\leq \theta$ is unsatisfiable, can not be relied upon, since $[\theta \mapsto$ dynamic] is a satisfiable substitution. The occurs check also fails for type inference on records that can contain as components other records and functions on records. We have extended the type inference algorithm to handle these problems, and we plan to present the results in the near future. 
Acknowledgements: David Watt and Peter Mosses contributed many helpful comments, and Tim Ramsey provided word processing expertise.

\section{References}

[1] Cardelli, L. A semantics of multiple inheritance. In LNCS 173: Semantics of Data Types, G. Kahn, et. al., eds., Springer, Berlin, 1984, pp. 51-68.

[2] Cardelli, L., and Mitchell, J. Records and subtypes. Proc. of 5th Conf. on Mathematical Foundations of Programming Semantics, New Orleans, LA, 1989, Springer LNCS, to appear.

[3] Cardelli, L., and Wegner, P. On understanding types, data abstraction, and polymorphism. Computing Surveys 17-4 (1985) 471-522.

[4] Even, S. An implementation of action semantics. M.S. report, Computer Science Dept., Iowa State Univ., Ames, Iowa, 1987.

[5] Even, S., and Schmidt, D.A. Category-sorted algebra-based action semantics. Proc. Conf. on Algebraic Methodology and Software Technology, Iowa City, IA, May 1989. To appear in Theoretical Computer Science.

[6] Fuh, Y.-C., and Mishra, P. Type inference with subtypes. In LNCS 300: ESOP '88, H. Ganzinger, ed., Springer, Berlin, 1988, pp, 94-114.

[7] . Polymorphic type inference: closing the theory-practice gap. In LNCS 351: TAPSOFT 89 , J. Diaz and F. Orejas, eds. Springer, Berlin, 1989.

[8] Jategaonkar, L., and Mitchell, J. ML with extended pattern matching and subtypes. Proc. 1988 ACM Conf. on LISP and Functional Programming, Snowbird, Utah, July 1988, pp. 198-211.

[9] Milner, R. A theory of type polymorphism in programming. J. of Computer and System Sci. 17 (1983) 267-310.

[10] Mitchell, J. Coercion and type inference. Proc. 11th ACM Symp. on Prin. of Prog. Lang., Salt Lake City, Utah, 1984, pp. 175-186.

[11] Mosses, P. Abstract semantic algebras! In Formal Description of Programming Concepts II, D. Bjoemer, ed., North-Holland, Amsterdam, 1983, pp. 45-72.

[12] _. A basic abstract semantic algebra. In LNCS 173: Semantics of data types, Springer, Berlin, 1984, pp. 87-108.

[13] _. The modularity of action semantics. To appear in SDF Benchmark Series in Computational Linguistics-Workshop II, MIT Press, Cambridge.

[14] _. Unified algebras and action semantics. In LNCS 349: STACS89, B. Monien, R. Cori, eds., Springer, Berlin, 1989.

[15] _ and Watt, D. The use of action semantics. In Formal Description of Programming Concepts III, North-Holland, Amsterdam, 1987.

[16] Ohori, A., and Buneman, P. Type inference in a database programming language. Proc. 1988 ACM Conf. on LISP and Functional Programming, Snowbird, Utah, 1988, pp.174-183.

[17] Remy, Didier. Typechecking records and variants in a natural extension of ML. In Proc. 16th ACM Symp. on Principles of Prog. Languages, Austin, Texas, 1989, pp. 77-88.

[18] Reynolds, J. Using category theory to design implicit conversions and generic operators. In LNCS 94: Semantics-Directed Compiler Generation, N. Jones, ed. Springer, 1980, pp. 211-258. 
[19] _. The essence of Algol. In Algorithmic Languages, J. deBakker and J.C. vanVliet, eds., Noth-Holland, Amsterdam, 1981, pp. 345-372.

[20] _.. Semantics as a design tool. Course lecture notes, Computer Science Dept., CamegieMellon Univ., Pittsburgh, PA, 1988.

[21] Robinson, J.A. A machine-oriented logic based on the resolution principle. J. ACM 12-1 (1965) 23-41.

[22] Stansifer, R. Type inference with subtypes. Proc. 15th ACM Symp. on Prin. of Prog. Lang., San Diego, CA, 1988, pp. 88-97.

[23] Tofte, M. Operational semantics and polymorphic type inference. Ph.D. thesis, Computer Science Dept., Edinburgh Univ., Edinburgh, Scotland, 1988.

[24] Wand, M. Type inference for record concatenation and multiple inheritance. Proc. 4th Symp. Logic in Computer Science, Asilomar, CA, 1989, IEEE Press.

[25] Watt, D. Executable semantic descriptions. Software: Practice and Experience, 16 (1986) 13-43.

[26] _.. An action semantics of standard ML. In LNCS 298: Mathematical Foundations of Programming Semantics, M. Main, et. al., eds., Springer, Berlin, 1987, pp. 572-598. 